absence of added GTP, and GTP-specific photolabelling of SRP54 could not be demonstrated in experiments that clearly demonstrate labelling of SRPR'. It may simply be that SRP54 comes equipped with a pre-bound GTP as it is isolated, but this crucial point needs to be established.

This reservation notwithstanding, a number of questions about the possible role played by GTP become interesting and important. As there are now apparently at least two GTP's involved, in what order and for what purposes are they hydrolysed? Is the SRP54 GTP hydrolyzed when a signal peptide is first bound to SRP as translation pauses? Is the second GTP, bound to SRPR, hydrolysed later when the SRP54-signal peptide complex is recognized by the receptor at the ER membrane? If so, as implied speculatively by Walter and colleagues ${ }^{3}$, GTP hydrolysis at both of these successive steps could be used to improve the fidelity of signal recognition. As with Tu in protein synthesis, the intrinsic GTPase rate of SRP and SRPR could serve as a pair of internal standards by which to measure the persistence of signal-peptide binding.

In one view, incorrectly matched (nonsignal) peptides would have an opportunity to dissociate from SRP54 before the switch of GTP hydrolysis is thrown, fixing the bound peptide in place and stopping elongation. The bound peptide could be further scrutinized in the context of its binding site on SRP54 (just as antigen peptides are recognized in the context of MHC molecules presenting them) by the next GTP-binding protein, SRPR. Only the most persistent peptide complexes, which would contain correct signal peptides, would proceed irreversibly forward in the translocation pathway when the GTPase timer runs out and the second GTP is hydrolysed, simultaneously powering the threading of the chain and the release of SRP. Two successive rounds of such kinetic filtration would prevent almost all errors of mistaken translocation.

As intriguing as the G-domain of SRP54 is the other, carboxy-terminal half of SRP54, termed the M-domain by Walter and colleagues ${ }^{3}$ because of its remarkably high content of encoded methionine. The M-domain is without a homologue in eukaryotes, but is highly homologous ${ }^{2,3}$ to an Escherichia coli protein of unknown function predicted from an open reading frame of DNA sequence. (Walter and colleagues name this sequence $f f h$, for fifty four homologue, which is appropriate as the predicted protein would be similar in size to SRP54 and would contain both its $\mathrm{G}$ - and $\mathrm{M}$-domains, although whether it performs a role in signal recognition in bacteria remains to be determined.)

Secondary structure algorithms applied to SRP54 and the $f f h$ M-domain predict $^{3}$ three distinct helices whose positions and boundaries coincide almost precisely in the two proteins, and a fourth helix found only in SRP54. As the primary sequences in these regions are only 38 per cent identical, this is a striking coincidence that lends some credence to the validity of the helix assignment. It is striking and of special interest that all four predicted helices are amphipathic. Hydrophobic residues, including all of the methionine residues, in the helical regions would be clustered exclusively on one face of each helix; patches of strongly polar residues would be found on the opposite face. Three of the four helices predicted for the M-domain of SRP54 have hydrophobic faces composed mainly of methionine; the fourth amphipathic helix would be methionine-free.

This suggested organization raises the intriguing possibility that the hydrophobic ER signal peptides are bound in a pocket lined by the hydrophobic faces of the putative amphipathic helices. With this in mind, Walter and colleagues propose an ingenious, but even more speculative, solution to the enigma of how signal peptides of diverse sequences and shapes can all be accommodated within the same binding site of SRP54. They point out that methionine is unique among the hydrophobic amino acids in that its side chain is unbranched; others (including leucine and isoleucine, which are similarly hydrophobic and frequently found to replace methionine in phylogenetic comparisons) have branched side chains with sterically constrained conformations. The more flexible methionine side chains are envisaged to project into the signal-peptidebinding groove like the flexible bristles of a brush, providing a plastic environment that can accommodate itself to a variety of different (though hydrophobic) primary sequences so as to maximize close packing.

Although attractive, such notions are highly speculative. The location of the signal-peptide-binding site within SRP54 has not even been determined (in fact, Dobberstein and colleagues ${ }^{2}$ suggest that peptide binding may occur within the $\mathrm{G}$-domain by analogy with the location of the effector region in $\mathrm{Tu}$ and ras proteins). Nonetheless, the seminal concept of a plastic environment that detects a predominant physical property is precisely what is needed to explain the kind of signal recognition that typically occurs in protein sorting. It may well be that methionine bristles will be added to zinc fingers and leucine zippers in the growing armoury of prostheses now employed in biology.

James E. Rothman is in the Department of Biology, Princeton University, Princeton, New Jersey 08544-1014, USA.

1. Connolly, T. \& Gilmore, R. Cell 57, 599-610 (1989).
2. Römisch, K. et al. Nature 340, 478-482 (1989).
3. Bernstein, H. et al. Nature 340, 482-486 (1989).
4. Thompson, R. Trends biochem. Sci. 13, 91-93 (1988).
5. Connolly, T. \& Gilmore, R. J. cell Biol. 103, 2253 (1986).
6. Walter, P. \& Lingappa, V.R. A. Rev. cell Biol. 2, 499 (1986).

\section{Base cunning}

THE roads of Britain are crumbling under the impact of the ever-heavier lorries permitted by European standards. Their relentless pounding overloads the road surface; ultimately it cracks, water gets in, and the surface breaks up. Even the best foundations yield to shock and pressure; every structure laid on the earth settles as the ground gradually compacts beneath it.

Now Daedalus has the answer. He points out that if you dig a hole, the excavated earth never fits neatly back into it. Digging has broken it up, and the resulting mass of loose and ill-fitting particles inevitably takes up more space than the original monolithic solid. Furthermore, the technology of bad concrete has made rapid strides in recent years. Additives like calcium chloride are known to embrittle it; whereas metallic iron in concrete can rust, expand and craze it. So DREADCO's engineers are devising the ultimate in bad and brittle concrete, loaded with salts, iron filings and so on. With this cheap and deplorable material as its foundation, even the most traffic-battered road should stay smooth and level. For instead of withstanding the vibration, the new road foundation will rapidly fragment. As a result, says Daedalus, it will use the force of the traffic against itself in a sort of road-maintenance Zen.

The surface of the road will be the usual slightly flexible, tarmac-topped structure. It transmits traffic vibration down to the new foundation concrete, which soon breaks up into a mass of small, close-fitting particles, rather like a shattered windscreen. Continuing traffic vibration shifts and tumbles these close-packed particles. Their positions are slowly randomized, their mutual close fit is lost, and the foundation steadily swells as its microstructure opens up. Like a soft mushroom pushing up a paving stone, the shattered but confined foundation lifts the road surface above it, exactly counteracting the slowly settling earth beneath. The road will remain flat and stable and safe. Thanks to DREADCO's new shoddy concrete, Britain's shoddy roads will effortlessly shake off the European heavyweight challenge.

Vibro-expansive concrete will be welcomed not merely by road builders, but by civil engineers of every kind. Urban buildings shaken by nearby traffic, factories full of vibrating machinery, railway lines and airport runways, all will be cheaper and more stable on their new crumbling foundations. Tottering cathedrals, trafficshaken monuments, and the like, will also benefit. Just inject the new concrete into the crucial gaps and cavities, and let the traffic do the rest. Daedalus has sent details of the process to the authorities at Pisa.

David Jones 